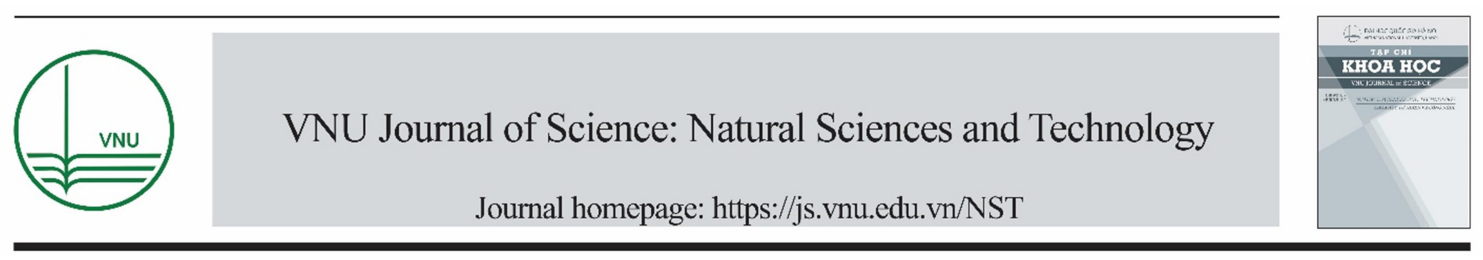

Original Article

\title{
The Isoelectric Point and the Surface Charge of Barium Titanate Nanoparticles/Graphene Oxide Determined Using the Electrophoretic Mobility Technique
}

\author{
Vuong Thi Vy Anh, Nguyen Thi Dung, Chu Ngoc Chau, Phan Thi Tuyet Mai, \\ Nguyen Xuan Hoan
}

VNU University of Science, 19 Le Thanh Tong, Hanoi, Vietnam

Received 15 August 2020

Revised 21 February 2021; Accepted 16 March 2021

\begin{abstract}
Barium titanate nanopowders, and composite materials of barium titanate/ graphene oxide (10 wt.\% of graphene oxide according to the initial composite composition) were synthesized by hydrothermal method at the fixed reaction condition of $200{ }^{\circ} \mathrm{C}$ and 24 hours. The obtained powders were characterized by different techniques: X-ray diffraction, FTIR spectroscopy, Particles size distribution, and Scanning electron microscopy. Zeta potential measurement under electrophoretic mobility technique was also employed to investigate the stability of the $\mathrm{BaTiO}_{3}$ nanoparticles and composite materials of barium titanate/graphene oxide. The results showed that the $\mathrm{BaTiO}_{3}$ present with the tetragonal crystal structure $(P 4 m m, a=$ $4.0000 \AA, c=4.0109 \AA$ ) and has uniform morphology with the grain sizes are in the range of 70 $140 \mathrm{~nm}$. The $\mathrm{BaTiO}_{3}$ nanoparticles were well distribution and covered on a surface of graphene oxide. The $\mathrm{BaTiO}_{3}$ nanoparticles, and $\mathrm{BaTiO}_{3}$ /graphene oxide are stable in alkali, neutral media, and acidic media up to $\mathrm{pH} \sim 5$.
\end{abstract}

Keywords: Graphene oxide, barium titanate, $\mathrm{BaTiO}_{3} / \mathrm{GO}$, Zeta potential, electrophoretic mobility.

${ }^{*}$ Corresponding author.

Email address: hoannx@vnu.edu.vn

https://doi.org/10. 25073/2588-1140/vnunst.5114 


\title{
Đặc trưng thế bề mặt và điểm đẳng điện của vật liệu nano $\mathrm{BaTiO}_{3} /$ graphen oxit bằng phương pháp điện di
}

\author{
Vương Thị Vy Anh, Nguyễn Thị Dung, Chu Ngọc Châu, Phan Thị Tuyết Mai, \\ Nguyễn Xuân Hoàn*
}

Trường Đại học Khoa học Tự nhiên, Đại học Quốc gia Hà Nội,

19 Lê Thánh Tông, Hà Nội, Việt Nam

Nhận ngày 15 tháng 08 năm 2020

Chỉnh sửa ngày 21 tháng 2 năm 2021; Chấp nhận đăng ngày 16 tháng 3 năm 2021

\begin{abstract}
Tóm tắt: Trong nghiên cứu này, vật liệu nano bari titanat và compozit bari titanat/graphen oxit ( $10 \%$ khối lượng graphen oxit) được chế tạo bằng phương pháp thủy nhiệt tại điều kiện $200{ }^{\circ} \mathrm{C} / 24$ giờ. Các vật liệu được đặc trưng các tính chất: nhiễu xạ tia $\mathrm{X}$, phổ hồng ngoại, phân bố kích cỡ hạt bằng tia Laser, chụp ảnh hiển vi điện tử quét. Phép đo thế Zeta được dùng để đánh giá độ bền phân tán của nano $\mathrm{BaTiO}_{3}$ và compozit bari titanat/graphen oxit, sử dụng kỹ thuật điện di. Kết quả thu được cho thấy, $\mathrm{BaTiO}_{3}$ thuộc cấu trúc mạng lưới tinh thể tứ phương $(P 4 m m, a=4,0000 \AA ⿻ 丿$ $4,0109 \AA$ ), và có độ đồng nhất cao về kích thước hạt trong khoảng $70-140 \mathrm{~nm}$. Các hạt nano $\mathrm{BaTiO}_{3}$ được phân tán đồng đều trên bề mặt của graphen oxit. $\mathrm{Hạt} \mathrm{BaTiO}_{3}$, và $\mathrm{BaTiO}_{3} /$ graphen oxit tồn tại bền trong cả môi trường kiềm và môi trường trung tính, ứng với $\mathrm{pH}>5$.
\end{abstract}

Tù khóa: Graphen oxit, bari titanat, $\mathrm{BaTiO}_{3} / \mathrm{GO}$, thế Zeta, điện di.

\section{Mở đầu}

Bari titanat $\left(\mathrm{BaTiO}_{3}\right)$ là vật liệu được quan tâm nghiên cứu do có các tính chất: điện môi, áp điện, sắt điện..., nên được định hướng sử dụng cho nhiều ứng dụng quan trọng trong ngành các công nghiệp điện, điện tử, cảm biến,...[1,2]. $\mathrm{BaTiO}_{3}$ được kết hợp để tạo vật liệu tổ hợp với các vật liệu khác góp phần mở rộng khả năng ứng dụng cho ắc quy liti-ion, siêu tụ điện $[3,4]$. Trong số đó, có thể kể đến việc tạo compozit của với các vật liệu cacbon như ống nano cacbon, graphen hay graphen oxit,...[5-8]; tiếp đó, chúng được phân tán/kết hợp với các vật liệu nền polyme để chế tạo các vật liệu polyme compozit đa chức năng $[9,10]$. Tính chất của vật liệu polyme compozit chế tạo phụ thuộc vào khả năng phân tán và độ bền của các hạt $\mathrm{BaTiO}_{3}$ trong môi trường phân tán [11].

\footnotetext{
Tác giả liên hệ.

Địa chỉemail: hoannx@vnu.edu.vn
}

https://doi.org/10.25073/2588-1140/vnunst.5114
Chính vì thế, nghiên cứu bề mặt, đặc biệt là thế bề mặt, cũng như xác định điểm đẳng điện (isoelectric point, IEP), hay điểm điện tích không (point of zero charge, $P Z C$ ) cho phép dự đoán khả năng phân tán, cũng như độ bền của hạt vật liệu trong môi trường phân tán. Hiện nay, mới chỉ có số ít nghiên cứu khảo sát trên hạt vật liệu $\mathrm{BaTiO}_{3}$ ở dạng cấu trúc lập phương, và các $\mathrm{BaTiO}_{3}$ pha tạp nguyên tố, hay graphen oxit $[12,13]$, nhưng gần như chưa có công bố nào đề cập đến vật liệu compozit $\mathrm{BaTiO}_{3} /$ graphen oxit. Trong nghiên cứu này, các vật liệu nano $\mathrm{BaTiO}_{3}$ ở dạng cấu trúc tinh thể tứ phương, vật liệu compozit tổ hợp $\mathrm{BaTiO}_{3} /$ graphen oxit đã được chế tạo trực tiếp bằng phương pháp thủy nhiệt trong môi trường kiềm của $\mathrm{KOH}$, và được sử dụng để nghiên cứu tính chất bề mặt, điểm đẳng điện trên cơ sở của phương pháp điện di.

\section{Thực nghiệm}

Hóa chất chính được sử dụng trong nghiên cứu gồm: $\mathrm{BaCl}_{2} \cdot 2 \mathrm{H}_{2} \mathrm{O} \quad(>99 \%, \quad$ Merck $)$, 
$\mathrm{TiCl}_{3}(15 \%$ trong $\mathrm{HCl}$, Merck), $\mathrm{HCl}(37 \%$, Merck), $\mathrm{KOH}$ ( $>82 \%$, Merck), và graphen oxit (GO) chế tạo theo phương pháp Hummer cải tiến [14], sử dụng các hóa chất tinh khiết gồm: graphit bột, $\mathrm{H}_{2} \mathrm{SO}_{4}, \mathrm{H}_{3} \mathrm{PO}_{4}, \mathrm{KMnO}_{4}$ và $\mathrm{H}_{2} \mathrm{O}_{2}$.

Vật liệu $\mathrm{BaTiO}_{3}$ được lựa chọn tổng hợp bằng phương pháp thuỷ nhiệt theo quy trình đã công bố [15] tại điều kiện: sử dụng hỗn hợp muối $\mathrm{Ba}^{2+}, \mathrm{Ti}^{3+}$ (tỷ lệ $\mathrm{Ba} / \mathrm{Ti}=1,6$ ), điều kiện phản ứng $200{ }^{\circ} \mathrm{C} / 24$ giờ.

Với vật liệu compozit $\mathrm{BaTiO}_{3} /$ graphen oxit (10 \% khối lượng graphen oxit) theo quy trình sau: Graphen oxit được phân tán siêu âm với lượng nước cất vừa đủ trong 15 phút, sau đó hỗn hợp muối $\mathrm{Ba}^{2+}, \mathrm{Ti}^{3+}$ được thêm vào dung dịch trên, và cuối cùng là dung dịch $\mathrm{KOH}$. Hỗn hợp các chất phản ứng được chuyển vào bình thủy nhiệt và thực hiện phản ứng trong cùng điều kiện như chế tạo vật liệu $\mathrm{BaTiO}_{3}$.

Sản phẩm $\mathrm{BaTiO}_{3}, \mathrm{GO}$, và $\mathrm{BaTiO}_{3} /$ graphen oxit tổng hợp được nghiên cứu các đặc tính và tính chất: Xác định pha bằng nhiễu xạ tia $\mathrm{X}$ trên thiết bị nhiễu xạ Bruker D8 Advance $\left(\lambda_{\mathrm{CuK} \alpha}=\right.$ $1,5406 \AA$, góc quét $2 \theta=0,03^{\circ} /$ step, khoảng quét từ $10-70^{\circ}$ ). Phổ hồng ngoại trên thiết bị Jasco FT/IR-6300, dải quét $4000-400 \mathrm{~cm}^{-1}$, sử dụng kỹ thuật ép viên $\mathrm{KBr}$. Đường cong phân bố cỡ hạt của vật liệu được xác định trên thiết bị phân bố cỡ hạt bằng tia Laser (Shimadzu SALD2101). Hình thái học hạt vật liệu được quan sát trên kính hiển vi điện tử quét phân giải cao NOVA NanoSEM.

Thế bề mặt và điểm đẳng điện của vật liệu $\mathrm{BaTiO}_{3}, \mathrm{BaTiO}_{3} /$ graphen oxit được đánh giá trên thiết bị Zeta Phoremeter IV (CAD Instrumen-tation), trên cơ sở phương pháp điện di, tại điện thế $100 \mathrm{~V}$. Mẫu vật liệu được phân tán trong môi trường nước cất chứa $0,001 \mathrm{M}$ $\mathrm{KCl}$ (với mục đích tăng độ dẫn và giữ lực ion không đổi), và khảo sát tại các giá trị $\mathrm{pH}$ khác nhau. Các dung dịch loãng $0,1 \mathrm{M} \mathrm{HCl}$, và $\mathrm{KOH}$ được dùng để điều chỉnh $\mathrm{pH}$ của môi trường. Gần đúng theo phương trình Schmoluchowski được sử dụng để tính giá trị điện thế bề mặt hạt (hay thế Zeta).

\section{Kết quả và thảo luận}

Hình 1a là giản đồ nhiễu xạ tia $\mathrm{X}$ của mẫu bột $\mathrm{BaTiO}_{3}, \mathrm{GO}$, và $\mathrm{BaTiO}_{3} /$ graphen oxit $\left(\mathrm{BaTiO}_{3} / \mathrm{GO}\right)$. Giản đồ XRD của mẫu $\mathrm{GO}$ chỉ xuất hiện duy nhất pic nhiễu xạ đặc trưng của GO tại vị trí góc nhiễu xạ $2 \theta=10,8^{\circ}[6,14]$.
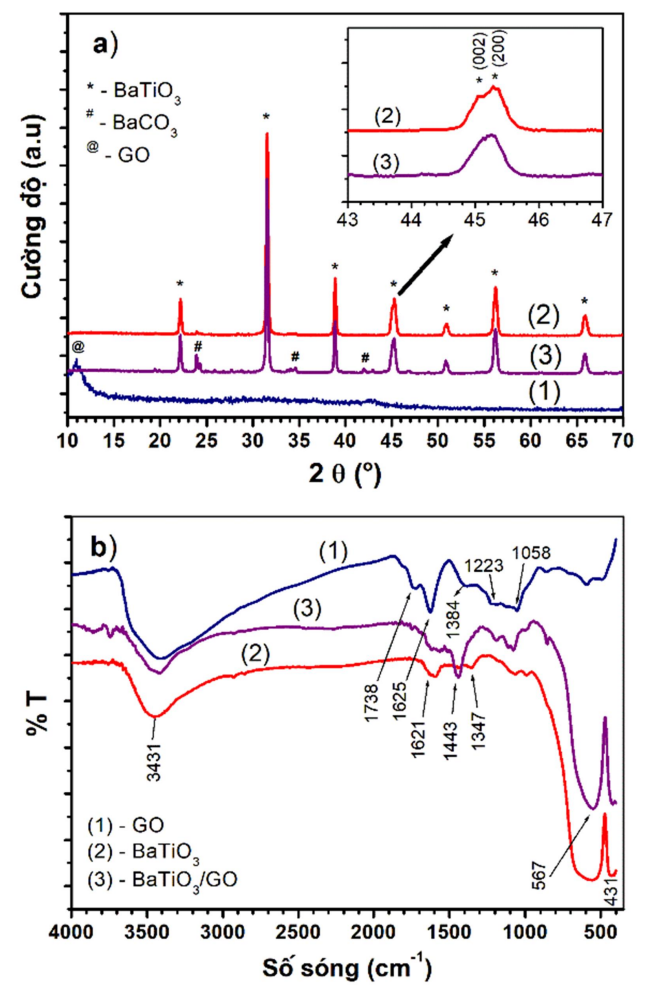

Hình 1. (a) - Giản đồ nhiễu xạ tia $X$ của các mẫu GO (1), $\mathrm{BaTiO}_{3}$ (2), và $\mathrm{BaTiO}_{3}$ /graphen oxit (3);

(b) - Phổ hồng ngoại tương ứng của vật liệu chế tạo.

Trên giản đồ nhiễu xạ của mẫu $\mathrm{BaTiO}_{3}$, chỉ xuất hiện chủ yếu các pic với cường độ lớn, đặc trưng cho pha vật liệu $\mathrm{BaTiO}_{3}$, thuộc hệ mạng lưới tinh thể tứ phương $(P 4 \mathrm{~mm}$, ICSD code: 01070-9164) $[15,16]$; được quan sát rõ khi phóng đại tại góc $2 \theta$ trong khoảng $45,2^{\circ}$ với sự phân tách thành 2 pic nhiễu xạ ứng với mặt phẳng (002) và $(200)$; và ở khoảng góc $56,1^{\circ}$, với sự phân tách 2 pic nhiễu xạ ứng với mặt phẳng (112) và (211). Bên cạnh đó, còn xuất hiện pic của pha $\mathrm{BaCO}_{3}$ (Pmcn, orthorhombic, ICSD code: 00-005-0378) với cường độ yếu, vì phản 
ứng thủy nhiệt được thực hiện trong môi trường $\mathrm{KOH}$. Tương tự như $\mathrm{BaTiO}_{3}$, mẫu $\mathrm{BaTiO}_{3} / \mathrm{GO}$ thu được với pha $\mathrm{BaTiO}_{3}$ có cấu trúc tứ phương tương ứng các mặt phẳng mạng tinh thể: (100), (101), (110), (111), (002), (200), (210), (112), (211), (202) và (220). Sử dụng phân tích Rietveld trên phần mềm PowderCell, cho phép tính các thông số mạng tinh thể thực nghiệm của $\mathrm{BaTiO}_{3}$; đối với mẫu vật liệu $\mathrm{BaTiO}_{3}: a=$ $4,0000 \AA ⿻, c=4,0109 \AA$, và đối với mẫu vật liệu $\mathrm{BaTiO}_{3} / \mathrm{GO}: a=4,0043 \AA, c=4,0089 \AA$, tương ứng.

Hình $1 \mathrm{~b}$ trình bày phổ hồng ngoại FT-IR của mẫu $\mathrm{GO}, \mathrm{BaTiO}_{3}, \mathrm{BaTiO}_{3} / \mathrm{GO}$. Phổ hồng ngoại của $\mathrm{GO}$ xuất hiện các dải sóng hấp thụ tai: $3431,1738,1625,1384,1223,1058 \mathrm{~cm}^{-1}$, ứng với các dao động đặc trưng của nhóm : $\mathrm{O}-$ $\mathrm{H}, \mathrm{C}=\mathrm{O}, \mathrm{C}-\mathrm{C}, \mathrm{C}-\mathrm{OH}$ và $\mathrm{C}-\mathrm{O}[8,9]$. Trên mẫu $\mathrm{BaTiO}_{3}$ và $\mathrm{BaTiO}_{3} / \mathrm{GO}$, dải sóng đặc trưng cho dao động $\mathrm{Ti}-\mathrm{O}$ trong cấu trúc titanat của $\mathrm{BaTiO}_{3}$ tương ứng với các vị trí số sóng có cường độ hấp thụ lớn tại 567 và $431 \mathrm{~cm}^{-1}$ [6,11], bên cạnh dao động nhóm cacbonat ($\mathrm{CO}_{3}{ }^{2-}$ ) với cường độ nhỏ còn lại trong mẫu tại $1443 \mathrm{~cm}^{-1}$. Ngoài ra, xuất hiện dải sóng tại 3431 và $1621 \mathrm{~cm}^{-1}$ đặc trưng cho dao động của nước hấp phụ và nhóm hydroxyl trên bề mặt hạt vật liệu. Sự có mặt của nhóm hydroxyl trên bề mặt được biết đến là nguyên nhân gây nên giá trị điện thế âm của hạt vật liệu được xác định qua phép đo thế Zeta (tại Hình 4).

Kết quả chụp phân bố cỡ hạt bằng tia Laser trên mẫu bột $\mathrm{BaTiO}_{3}, \mathrm{GO}$ và $\mathrm{BaTiO}_{3} / \mathrm{GO}$ khi phân tán trong môi trường phân tán nước cất với tác nhân trợ phân tán $\mathrm{Na}\left(\mathrm{PO}_{3}\right)_{6}$ cùng ảnh chụp SEM trình bày trên Hình 2 và Hình 3 . Giá trị kích thước các hạt $\mathrm{BaTiO}_{3}$ nằm trong khoảng phân bố từ $70-140 \mathrm{~nm}$ với giá trị trung bình hạt là $95 \mathrm{~nm}$. Phân bố về kích thước hạt của $\mathrm{BaTiO}_{3}$ chỉ có một đơn pic nhọn thu được trên đường cong phân bố cho phép khẳng định sự đồng nhất về kích thước hạt vật liệu. Với mẫu vật liệu compozit $\mathrm{BaTiO}_{3} / \mathrm{GO}$, đường cong phân bố mở rộng tập trung trong dải kích thước từ $70 \mathrm{~nm}$ đến 1 micromet, một lượng nhỏ vật liệu tìm thấy với phân bố ở kích thước lớn trong khoảng 1 - 20 micromet. Kết quả thu được này tương đối phù hợp với hàm lượng $\mathrm{GO}-10 \%$ được phân tán trong quá trình tổng hợp thủy nhiệt để tạo compozit $\mathrm{BaTiO}_{3} / \mathrm{GO}$, khi đối chiếu với dải phân bố rộng từ 1 đến $50 \mu \mathrm{m}$ của mẫu GO (Hình 2b).
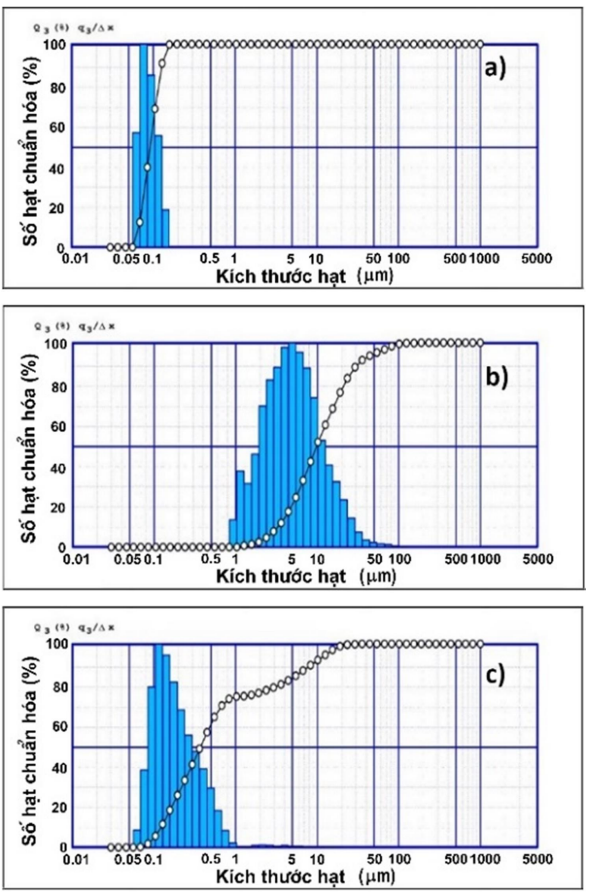

Hình 2. Giản đồ phân bố cỡ hạt của (a) $-\mathrm{BaTiO}_{3}$, (b) - GO, (c) - $\mathrm{BaTiO}_{3} / \mathrm{GO}$.

Các kết quả thu được quan sát từ ảnh chụp SEM của vật liệu tương đối phù hợp với kết quả thu được từ các đường cong phân phố về kích thước hạt đã chỉ ra trên Hình 2. Các hạt $\mathrm{BaTiO}_{3}$ có dạng gần cầu với kích thước hạt trong khoảng $90 \mathrm{~nm}$. Trên mẫu vật liệu compozit $\mathrm{BaTiO}_{3} / \mathrm{GO}$, quan sát thấy các hạt $\mathrm{BaTiO}_{3}$ có xu hướng bám - bao phủ trên bề mặt các tấm graphen oxit với kích thước hạt tương đồng như mẫu $\mathrm{BaTiO}_{3}$ (Hình $3 \mathrm{c}$ ), và so sánh cùng với ảnh chụp hình thái bề mặt mẫu bột graphen oxit tìm thấy ở dạng các tấm/ phiến mỏng (Hình 3b). 

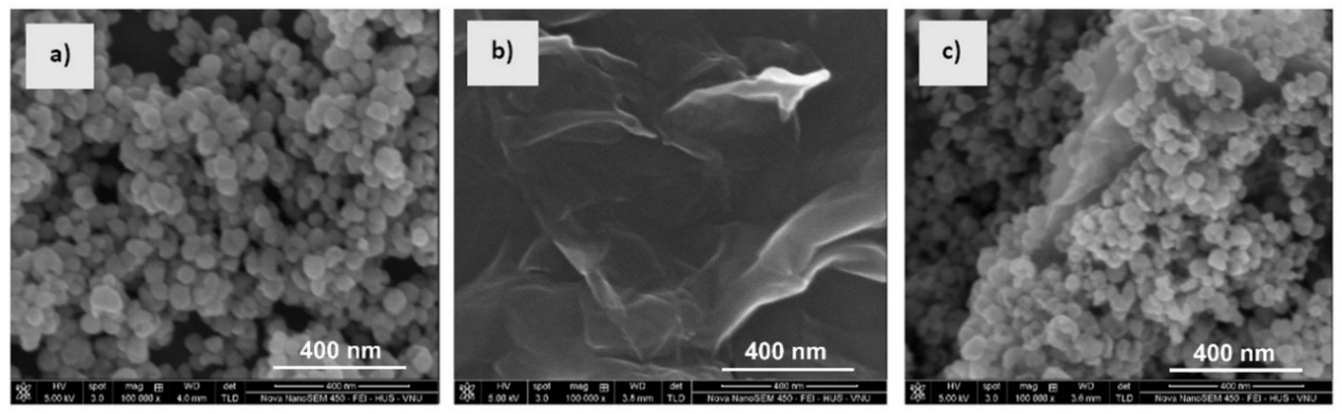

Hình 3. Ảnh chụp SEM của vật liệu: (a) - $\mathrm{BaTiO}_{3}$, (b) - $\mathrm{GO}$ và (c) - $\mathrm{BaTiO}_{3} / \mathrm{GO}$, tương ứng.
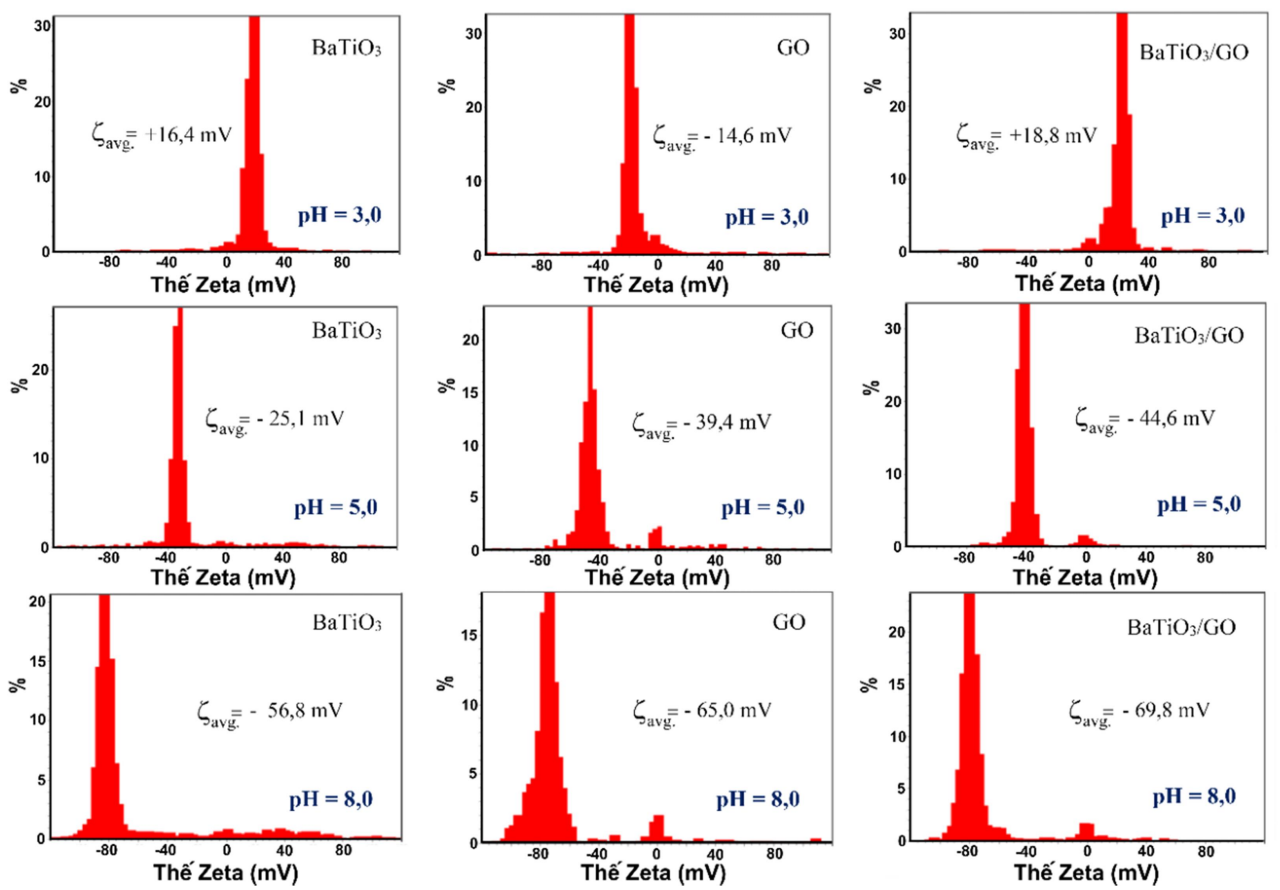

Hình 4. Giản đồ phân bố điện thế bề mặt của các hạt vật liệu $\mathrm{BaTiO}_{3}, \mathrm{GO}$ và $\mathrm{BaTiO}_{3} / \mathrm{GO}$ đo tại giá trị $\mathrm{pH}=3,5$, và 8 được phân tán trong dung dịch điện ly $\mathrm{KCl} 0,001 \mathrm{M}$.

Điện thế bề mặt hạt vật liệu $\mathrm{BaTiO}_{3}, \mathrm{GO}$ và $\mathrm{BaTiO}_{3} / \mathrm{GO}$ được khảo sát trong môi trường $\mathrm{pH}$ khác nhau chứa $0,001 \mathrm{M} \mathrm{KCl}$. Tại mỗi giá trị $\mathrm{pH}$ tương ứng, mẫu được đo trung bình từ 15 đến 20 lần để xác định giá trị thế Zeta trung bình, và từ đó xây dựng đường cong phân bố về điện thế bề mặt hạt. Trên Hình 4 giới thiệu đại diện giản đồ phân bố thế Zeta của các mẫu tại 3 giá trị $\mathrm{pH}$ khảo sát, lần lượt bằng $\mathrm{pH}=3,5$ và 8. Tại giá trị $\mathrm{pH}=5$, các hạt vật liệu chế tạo đều có điện thế âm trên bề mặt, giá trị thế Zeta trung bình lần lượt bằng: $-25,1 \mathrm{mV},-39,4 \mathrm{mV}$ và $-44,6 \mathrm{mV}$; với các giá trị điện thế âm trên bề mặt, chứng tỏ các hạt nano $\mathrm{BaTiO}_{3}, \mathrm{GO}$, và $\mathrm{BaTiO}_{3} / \mathrm{GO}$ chế tạo bằng phương pháp thủy nhiệt đều bền trong môi trường phân tán dung 
môi nước. Giá trị âm về thế bề mặt của các hạt $\mathrm{BaTiO}_{3}$ có thể lý giải trên bề mặt có chứa các nhóm $\mathrm{OH}^{-}$dư và mang điện âm đã tạo liên kết với bề mặt các hạt theo mô hình: (bề mặt hạt) \{$\mathrm{O}-\mathrm{H}-\}_{\mathrm{n}}[11]$. Các đường phân bố về điện thế bề mặt trên các mẫu đều có dạng đơn pic phân tán hoàn toàn phù hợp với kết quả thu được trên đường phân bố về kích thước hạt như đã chỉ ra trên Hình 2. Sự dịch chuyển giá trị điện thế bề mặt của từng mẫu được quan sát rõ nét khi thay đổi từ $\mathrm{pH}=3$ sang giá trị $\mathrm{pH}=8$, với xu hướng dịch chuyển về phía điện thế âm hơn theo chiều tăng của $\mathrm{pH}$ của môi trường. Hình 5 biễu diễn sự phụ thuộc của thế Zeta vào $\mathrm{pH}$ dựa trên giá trị trung bình thế Zeta của các mẫu tại mỗi giá trị $\mathrm{pH}$ khảo sát.

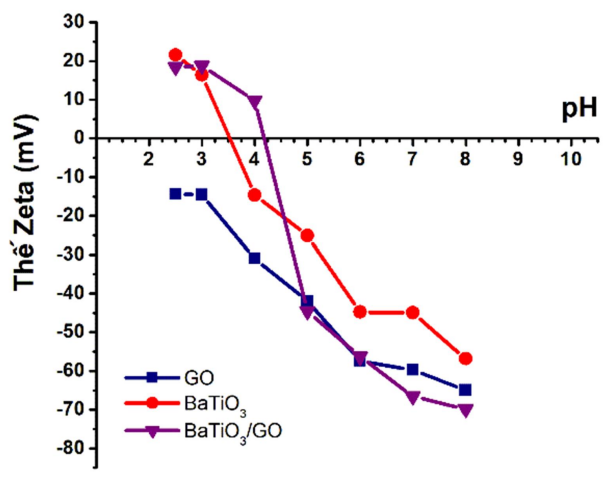

Hình 5. Sự phụ thuộc của điện thế bề mặt vào $\mathrm{pH}$ của các hạt vật liệu $\mathrm{BaTiO}_{3}, \mathrm{GO}$ và $\mathrm{BaTiO}_{3} / \mathrm{GO}$ được phân tán trong dung dịch điện ly $\mathrm{KCl}$.

Từ đồ thị Hình 5 cho thấy, với mẫu GO, giá trị điện thế bề mặt hạt luôn âm trong khoảng $\mathrm{pH}$ khảo sát từ 2,5 đến 8 . Kết quả thu được này tương đồng với các kết quả trong nghiên cứu [13]. Khi $\mathrm{pH}$ tăng, giá trị điện thế bề mặt càng giảm mạnh về phía vùng điện thế âm hơn. GO có xu hướng bền phân tán khi $\mathrm{pH}>4$. Giá trị điện thế bề mặt âm của $\mathrm{GO}$, đã minh chứng cho khả năng hấp phụ các ion $\mathrm{Ba}^{2+}$ và $\mathrm{Ti}^{3+}$ nhờ tương tác tĩnh điện. Từ đó, các hạt $\mathrm{BaTiO}_{3}$ hình thành trong quá trình tổng hợp thủy nhiệt dễ dàng phân tán/bám dính đều trên bề mặt $\mathrm{GO}$ (ảnh chụp $\mathrm{SEM}$ - Hình $3 \mathrm{c}$ ). Các mẫu $\mathrm{BaTiO}_{3}$ và $\mathrm{BaTiO}_{3} / \mathrm{GO}$ khi phân tán trong dung dịch chất điện ly $0,001 \mathrm{M} \mathrm{KCl}$ có giá trị thế Zeta không khác biệt nhiều và đều có giá trị điểm đẳng điện (IEP hoặc $P Z C$ ) tại $\mathrm{pH}$ trong khoảng từ 3,5 đển 4,2 . So sánh với các kết quả đã công bố trên hạt $\mathrm{BaTiO}_{3}$ khi chỉ phân tán trong môi trường nước cất cho giá trị $\mathrm{pH}_{P Z C} \approx 2,4$, khẳng định sự có mặt của dung dịch điện ly đã làm dịch chuyển giá trị điểm đẳng điện về phía $\mathrm{pH}$ cao hơn như đã chỉ ra trong các nghiên cứu $[12,17]$. Ở vùng $\mathrm{pH} \sim 5$, độ lớn của giá trị thế Zeta, $|\zeta|>25 \mathrm{mV}$ và trong khoảng $\mathrm{pH}>6$, độ lớn giá trị thế Zeta của các mẫu gần như không thay đổi, $|\zeta|>40$ $\mathrm{mV}$. Giá trị này âm hơn nhiều so với các nghiên cứu đã công bố và cho thấy các hạt $\mathrm{BaTiO}_{3}$ và $\mathrm{BaTiO}_{3} / \mathrm{GO}$ chế tạo bằng phương pháp thủy nhiệt tồn tại bền trong môi trường phân tán trung tính và kiềm. Trong vùng giá trị $\mathrm{pH}<3,0$ (môi trường axit), các hạt nano $\mathrm{BaTiO}_{3}$, hoặc pha tạp chất $\mathrm{BaCO}_{3}$ đã bị hòa tan một phần trên bề mặt, giải phóng các ion $\mathrm{Ba}^{2+}$ ra môi trường $[12,17]$ và được hấp phụ ngược trở lại, kết quả là giá trị thế Zeta của $\mathrm{BaTiO}_{3}$ đã dịch chuyển từ vùng giá trị âm, $\zeta \sim-9,3 \mathrm{mV}$, tại $\mathrm{pH}=4$ sang vùng có giá trị điện thế dương hơn $\zeta \sim+18,8$ $\mathrm{mV}$, tại $\mathrm{pH}=3$. Kết quả tương tự cũng được quan sát trên mẫu compozit $\mathrm{BaTiO}_{3} / \mathrm{GO}$.

\section{Kết luận}

Đã chế tạo vật liệu nano $\mathrm{BaTiO}_{3}$ và $\mathrm{BaTiO}_{3} / \mathrm{GO}(10 \% \mathrm{GO}$ về khối lượng) bằng phương pháp tổng hợp thủy nhiệt. Các kết quả thu được thông qua các phương pháp đặc trưng tính chất cho thấy $\mathrm{BaTiO}_{3}$ tồn tại trong cấu trúc tinh thể tứ phương $(P 4 m m, a=4,0000 \AA, c=$ 4,0109 $\AA$ ). Vật liệu $\mathrm{BaTiO}_{3} / \mathrm{GO}$ với các hạt $\mathrm{BaTiO}_{3}$ được phân tán đều trên bề mặt $\mathrm{GO}$, có hình thái học khá đồng nhất so sánh với kích thước của $\mathrm{BaTiO}_{3}$, kích thước hạt $100 \mathrm{~nm}$. Sử dụng phương pháp đo thế Zeta, sử dụng kỹ thuật điện di, để khảo sát điện thế bề mặt của các hạt $\mathrm{BaTiO}_{3}, \mathrm{BaTiO}_{3} / \mathrm{GO}$, và độ bền của các hạt tại các giá trị $\mathrm{pH}$ khác nhau cho thấy chúng đều tồn tại bền trong môi trường phân tán chứa chất điện ly $0,001 \mathrm{M} \mathrm{KCl}$ khi $\mathrm{pH}>5$. Điểm đẳng điện của các hạt vật liệu $\mathrm{BaTiO}_{3}$ và $\mathrm{BaTiO}_{3} / \mathrm{GO}$ chế tạo trong nghiên cứu này cũng được xác định tương ứng với giá trị $\mathrm{pH}_{\mathrm{PZC}} \sim 3,5$ và 4,2 . 


\section{Lời cảm ơn}

Công trình này được hỗ trợ kinh phí nghiên cứu từ Quỹ Phát triển Khoa học và Công nghệ Quốc gia Việt Nam (NAFOSTED, mã số 104.06-2018.328).

\section{Tài liệu tham khảo}

[1] K. Byrappa, M. Yoshimura, Handbook of Hydrothermal Technology: Materials and Processing Technology. William Andrew, $2^{\text {nd }}$ ed, 754-773, (2012).

[2] S. Selvarajan, N.R. Alluri, A. Chandrasekhar, S.J. $\mathrm{Kim}, \mathrm{BaTiO}_{3}$ nanoparticles as biomaterial film for self-powered glucose sensor application, Sens. Actuators B Chem 234 (2016) 395-403. https://doi.org/10.1016/j.snb.2016.05.004.

[3] P. Sardarian, H. Naffakh-Moosavy, S.S.S. Afghahi, A newly-designed magnetic/dielectric $\left[\mathrm{Fe}_{3} \mathrm{O}_{4} / \mathrm{BaTiO}_{3} @ \mathrm{MWCNT}\right]$ nanocomposite system for modern electromagnetic absorption applications, J. Magn. Magn. Mater 441 (2017) 257-263. https://doi.org/10.1016/j.jmmm.2017.05. 074.

[4] S. Rajendran, K. Kesavan, R. Nithya, M. Ulaganathan, Transport, structural and thermal studies on nanocomposite polymer blend electrolytes for Li-ion battery applications, Curr. Appl. Phys 12 (2012) 789-793. https://doi.org/10. 1016/j.cap.2011.11.006.

[5] C. Mallada, J.L. Menéndez, O.J. Dura, M.A. López de la Torre, R. Menéndez, R. Santamaría, Spark plasma sintered $\mathrm{BaTiO}_{3} /$ graphene composites for thermoelectric applications, J. Eur. Ceram. Soc $37 \quad$ (2017) 3741-3746. https://doi.org/10.1016/j.jeurceramsoc.2017.02.02 7.

[6] J. Ran, M. Guo, L. Zhong, H. Fu, In situ growth of $\mathrm{BaTiO}_{3}$ nanotube on the surface of reduced graphene oxide: A lightweight electromagnetic absorber, J. Alloys Compd 773 (2019) 423-431. https://doi.org/10.1016/j.jallcom.2018.09.142.

[7] M. Sohail, M.S. Khan, N. Saeed, M. Arif, M. Irfan, M. Omer, Synthesis, structural, thermal and dielectric properties of graphene oxide based barium titinate composite films: Possible materials for embedded capacitors, Materials Discovery 10 (2017) 29-36. https://doi.org/10.101 6/j.md.2018. 04.001.

[8] Y. Zhao, X. Zhang, J. Liu, C. Wang, J. Li, H. Jin, Graphene oxide modified nano-sized $\mathrm{BaTiO}_{3}$ as photocatalyst, Ceram. Int 44 (2018) 15929-15934. https://doi.org/10.1016/j.ceramint.2018.06.013.
[9] J.H. Yang, X. Xie, Z.Z. He, Y. Lu, X.D. Qi, Y. Wang, Graphene oxide-tailored dispersion of hybrid barium titanate@polypyrrole particles and the dielectric composites, Chem. Eng. J 355 (2019) 137-149. https://doi.org/10.1016/j.cej.20 18.08.152

[10] H. Luo, Z. Wu, X. Zhou, Z. Yan, K. Zhou, D. Zhang, Enhanced performance of P(VDF-HFP) composites using two-dimensional $\mathrm{BaTiO}_{3}$ platelets and graphene hybrids, Compos. Sci. Technol 160 (2018) 237-244. https://doi.org/ 10.1016/j.compscitech.2018.03.034.

[11] T.T.M. Phan, N.C. Chu, V.B. Luu, H. Nguyen Xuan, D.T. Pham, I. Martin, P. Carrière, Enhancement of polarization property of silanemodified $\mathrm{BaTiO}_{3}$ nanoparticles and its effect in increasing dielectric property of epoxy/ $/ \mathrm{BaTiO}_{3}$ nanocomposites, Journal of Science: Advanced Materials and Devices 1 (2016) 90-97. https://doi.org/10.1016/j.jsamd.2016.04.005.

[12] M.C. Blanco López, B. Rand, F.L. Riley, The isoelectric point of $\mathrm{BaTiO}_{3}$, J. Eur. Ceram. Soc 20 (2000) 107-118. https://doi.org/10.1016/S09552219(99)00137-5.

[13] F. Baskoro, C.B. Wong, S.R. Kumar, C.W. Chang, C.H. Chen, D.W. Chen, S.J. Lue, Graphene oxide-cation interaction: Inter-layer spacing and zeta potential changes in response to various salt solutions, J. Membr. Sci 554 (2018) 253-263. https://doi.org/10.1016/j.memsci.2018. 03.006 .

[14] T.H. Le, S.D. Dao, H. Nguyen Xuan, H.T. Nguyen, X.V. Nguyen, Synthesis of $\mathrm{Fe}_{3} \mathrm{O}_{4}$ reduced graphene oxide modified tissue-paper and application in the treatment of methylene blue, VNU Journal of Science: Natural Sciences $\begin{array}{llll}\text { and } & \text { (2019) 5echnology } 35-63 .\end{array}$ https://doi.org/10. 25073/2588-1140/vnunst.4883.

[15] N.X. Hoan, C.N. Chau, Effect of the reaction temperature on the crystal structure and stability of the nano barium titanate prepared using hydrothermal synthesis, Vietnam Journal of Chemistry 51(6ABC) (2013) 558-562 (in Vietnamese).

[16] Inorganic Crystal Structure Database (ICSD) (C) 2020 FIZ Karlsruhe $\mathrm{GmbH}$, https://icsd.pro ducts.fiz-karlsruhe.de/. (accessed 10 August 2020)

[17] C.W. Chiang, J.H. Jean, Effects of barium dissolution on dispersing aqueous barium titanate suspensions, Mater. Chem. Phys 80 (2003) 647655. https://doi.org/10.1016/S0254-0584(03)000 88-9. 